https://doi.org/10.15407/ujpe65.9.802

O. BAKAI, M. BRATCHENKO, S. DYULDYA

National Science Center Kharkiv Institute of Physics and Technology, Nat. Acad. of Sci. of Ukraine

(1, Akademichna Str., Kharkiv 61108,Ukraine; e-mail: bakai@kipt.kharkov.ua)

\title{
ON THE SINGULARITY OF THE LIQUID-GAS COEXISTENCE CURVE DIAMETER ${ }^{1}$
}

\begin{abstract}
A simplified Anisimov-Wang variant of the complete scaling approach makes it possible to determine the amplitudes of singularities for the diameter of the phase coexistence curve (CXC) on the basis of the coefficients in the power series expansion of the mean-field free energy in the reduced temperature and pressure near the critical point. This method is applied to obtain the amplitudes for the leading critical singularities of the CXC diameter in the case of a fluid described in the framework of the mesoscopic mean-field model. The results obtained demonstrate that the amplitudes of leading singularities of the CXC diameter are determined by the mesoscopic asymmetry parameters of the heterophase fluid.
\end{abstract}

Keywords: gas-liquid coexistence curve, diameter singularity, heterophase fluctuations, mesoscopic model, heterophase fluid, mesoscopic asymmetry parameters.

\section{Introduction}

Experimental studies [1-5] testify that the gas-liquid coexistence curve (CXC) is not symmetric in the density, $\rho$, versus temperature, $T$, plane, and its diameter

$\rho_{d}=\frac{\rho_{v}^{\mathrm{CXC}}+\rho_{l}^{\mathrm{CXC}}}{2 \rho_{\mathrm{c}}}$

is singular at the critical point $\left(\rho_{\mathrm{c}}, T_{\mathrm{c}}\right)$. Here, $\rho_{v}^{\mathrm{CXC}}$ and $\rho_{l}^{\mathrm{CXC}}$ denote the densities of vapor (the subscript $v$ ) and liquid (the subscript $l$ ) at the $\mathrm{CXC}$, and $\rho_{\mathrm{c}}$ means the system density at the critical point.

The critical-point theory developed by applying the Kadanoff-Wilson renormalization group method [5-7] to the Ising model or the effective GinzburgLandau Hamiltonian [6-8] allows the critical indices of the order parameter and the correlation length to be calculated with high accuracy. Similar results were obtained by applying the method of collective variables [9].

The Lee-Yang lattice gas model [10] reduces the theory of a gas-liquid critical point to the Ising model with a scalar or vector order parameter, thus indicating that the critical point of the fluid belongs to the Ising universality class. Since the CXC in the Ising model is symmetric, its diameter is not singular. The singularity in the diameter arises owing to

(C) O. BAKAI, M. BRATCHENKO, S. DYULDYA, 2020 the presence of asymmetric components in the effective Hamiltonian. As a result, the method of scaling transformations and renormalization group has to be revisited.

The method of "complete scaling" (CS) formulated by Fisher et al. $[11,12]$ is an important step while studying the criticality of fluids. On the other hand, the method of "simplified complete scaling" (SCS) developed by Anisimov and Wang [13, 14] reduces the estimation of the amplitudes of the leading singular terms in the expression for the CXC diameter to the determination of only two of nine coefficients of the linear mixing of physical fields that are required in the CS approach. In Ref. [14], the values of those two coefficients were expressed in terms of the coefficients in the expansion of the mean-field chemical potential of a fluid into a double Taylor series in deviations of the temperature $T$ and the density $\rho$ from their values at the critical point $-T_{\mathrm{c}}$ and $\rho_{\mathrm{c}}$, respectively. It is this approach that we apply to the mesoscopic mean-field model of a fluid [15].

In Section 2, the results obtained for the CXC diameter in the framework of the complete $[11,12]$ and simplified complete $[13,14]$ scaling models are briefly recalled. In Section 3, the main ideas of the mesoscopic mean-field theory of a fluid [15] are pre-

1 This article is dedicated to the 75th anniversary of Academician L.A. Bulavin.

ISSN 2071-0194. Ukr. J. Phys. 2020. Vol. 65, No. 9 
sented. In Section 4, the governing parameters of the simplified complete scaling are determined in terms of the mesoscopic fluid theory, and the results obtained in Ref. [14] are ordered. The final section 5 is devoted to the discussion and conclusions.

\section{Simplified Complete Scaling Method for Determining the Amplitudes of the Leading CXC Singularities}

In the CS approach $[11,12]$, three Ising scaling fields are regarded to be linear functions of the reduced chemical potential $\hat{\mu}$, temperature $\tau$, and pressure $\pi$,

$\hat{\mu}=\frac{\mu-\mu_{\mathrm{c}}}{k_{\mathrm{B}} T_{\mathrm{c}}}, \quad \tau=\frac{T-T_{\mathrm{c}}}{T_{\mathrm{c}}}, \quad \pi=\frac{P-P_{\mathrm{c}}}{P_{\mathrm{c}}}$,

where $k_{\mathrm{B}}$ is the Boltzmann constant. In the framework of this approach, the following expression was obtained for the CXC diameter:

$\rho_{d}=1+D_{2 \beta}|\tau|^{2 \beta}+D_{1-\alpha}|\tau|^{1-\alpha}+D_{1}|\tau|+\ldots$.

Here, $\beta \approx 0.326$ and $\alpha \approx 0.109$ are universal critical indices. The amplitudes $D_{2 \beta}, D_{1-\alpha}$, and $D_{1}$ depend on nine coefficients of the linear mixing of the physical fields $\hat{\mu}, \tau$, and $\pi$. These coefficients are not universal and have to be determined from the analysis of experimental data.

Anisimov and Wang [13, 14] showed the compatibility of the CS approach and the mean-field approximation. They proposed the SCS method by having proved that the amplitudes of the singular and linear terms in Eq. (3) can be determined knowing only two field-mixing coefficients, $a_{3}$ and $b_{2}$. In terms of those coefficients, the expression for the CXC diameter looks like

$\rho_{d}=1+\frac{a_{3}}{1+a_{3}} B_{0}^{2}|\tau|^{2 \beta}-$

$-b_{2}\left(\frac{A_{0}^{-}}{1-\alpha}|\tau|^{1-\alpha}+B_{\mathrm{cr}}|\tau|\right)+\ldots$.

Here, the parameter $B_{0}$ can be found from the experimentally measured CXC near the critical point:

$\hat{\rho}_{\mathrm{CXC}}=\frac{\rho_{\mathrm{CXC}}-\rho_{\mathrm{c}}}{\rho_{\mathrm{c}}}= \pm B_{0}|\tau|^{\beta}+\ldots$.

The parameters $A_{0}^{-}$and $B_{\text {cr }}$ determine the critical specific (per molecule) heat capacity at a constant volume in the two-phase region, $C_{V}$,

$\frac{C_{V}}{k_{b}}=A_{0}^{-}|\tau|^{-\alpha}-B_{\mathrm{cr}}+\ldots$.

ISSN 2071-0194. Ukr. J. Phys. 2020. Vol. 65, No. 9
They can be found from experimental data. The coefficients $a_{3}$ and $b_{2}$ remain free parameters, which are determined by fitting expression (4) to an experimentally measured dependence of the CXC diameter on $\tau$.

Since the CXC asymmetry and the singularity of the CXC diameter are determined by the interaction of molecules, the coefficients $a_{3}$ and $b_{2}$ are ordered according to the "interaction volume" parameter $\varpi^{*}=\left(2 \xi_{0}^{+}\right)^{3} \rho_{\mathrm{c}}$, where $\xi_{0}^{+}$is the amplitude of the density correlation length in the single-phase region above the critical temperature $[14,16]$. Anisimov and Wang showed that the values of $a_{3}$ and $b_{2}$ are arranged along certain master curves $a_{3}\left(\varpi^{*}\right)$ and $b_{2}\left(\varpi^{*}\right)$ with acceptable deviations from them. The characteristic lengths $2 \xi_{0}^{+}$are comparable with the molecular size, and the parameter $\varpi^{*}$ acquires values of about $10^{-1}$. Therefore, $\varpi^{*}$ is an averaged microscopic parameter of molecular interactions.

In the mean-field model of a fluid, the CXC asymmetry appears owing to those terms in the effective Hamiltonian that violate its symmetry. In the case of the Ising-type Hamiltonians with a one-component scalar order parameter, the chemical potential can be represented as a double Taylor series in $\hat{\rho}$ and $\tau$,

$$
\begin{aligned}
& \hat{\mu}(\hat{\rho}, \tau)=\sum_{m, n=0}^{\infty} \frac{1}{m ! n !} \mu_{m n} \hat{\rho}^{m} \tau^{n}, \\
& \mu_{m n}=\left(\frac{\partial^{m+n} \hat{\mu}}{\partial^{m} \hat{\rho} \partial^{n} \tau}\right)_{\rho_{\mathrm{c}}, T_{\mathrm{c}}} .
\end{aligned}
$$

In Refs. [13, 14], the following representations for the parameters $a_{3}$ and $b_{2}$ in terms of the coefficients $\mu_{m n}$ were found:

$\frac{a_{3}}{1+a_{3}}=\frac{2 \mu_{21}}{3 \mu_{11}}-\frac{\mu_{40}}{5 \mu_{30}}, \quad b_{2}=\frac{1}{\mu_{11}}\left(\frac{\mu_{21}}{\mu_{11}}-\frac{\mu_{40}}{5 \mu_{30}}\right)$.

The coefficients $a_{3}$ and $b_{2}$ determined in Ref. [14] for some mean-field models are located near the master curves $a_{3}\left(\varpi^{*}\right)$ and $b_{2}\left(\varpi^{*}\right)$.

Proceeding to the consideration of the mean-field mesoscopic model of a heterophase fluid [15], we note that the coefficients in series (7), which generate the singularity of the CXC diameter, become functions of the asymmetry parameters. This circumstance allows the amplitudes of the singular components of the CXC diameter to be mutually related and ordered in terms of those parameters. 


\section{Mesoscopic Theory of Heterophase Fluid}

As follows from the Frenkel theory $[17,18]$ (see also review [19]), the fraction of molecules belonging to heterophase fluctuations (vapor bubbles in liquid and droplets in vapor) is small in the biphasic region far from the critical point (approximately at $\tau<\tau_{\text {cross }} \approx$ $\approx-0.18)$. At $\tau>\tau_{\text {cross }}$, there is a region near the critical point in the $(P, T)$-plane, where the gas and liquid fractions of the fluid form infinite and mutually penetrating (percolating) clusters. This is a region of strong heterophase fluctuations (SHFs). It also includes the region of critical fluctuations, the temperature width of which is about $10^{-2} T_{\mathrm{c}}$.

Kadanoff applied the hypothesis of scale invariance and the method of block-spin transformations to the effective Ising Hamiltonian [6]. Later, when substantiating the applicability of his theory to describe the gas-liquid critical point, he noted that the fluid is heterophase in the critical region:

"In the neighborhood of a critical point, a fluid shows a very special behavior. As one gets closer to the critical point, fluctuations appear that are almost, but not quite, like the ones found in the boiling region of the first-order transition. 〈...〉 The fluid has bubbles of liquid inside bubbles of vapor, which are themselves inside bubbles of liquid, almost ad infinitum. The size of the smallest bubbles is usually of the order of the range of intermolecular forces or the distance between molecules" [20].

Hence, Kadanoff considered that the smallest bubbles and droplets should be mesoscopic in size because a single molecule cannot be identified as belonging to a bubble or a droplet. Concerning the effective Hamiltonian for the fluid, Kadanoff saw its prototype in the lattice gas model developed by Lee and Yang [10]. This model, in such a way, reduced the theory of a gas-liquid critical point to the Hamiltonian of the Ising model. When proposing to replace atoms and voids in the Lee-Yang theory with droplets and bubbles, Kadanoff evidently stressed the necessity to preserve the applicability of block-spin transformations near the vapor-liquid critical point and to ignore both the transition to mesoscopic block-spin transformations and the absence of the CXC asymmetry in the Ising model.

The mesoscopic mean-field theory of heterophase fluid in the SHF region was formulated in Ref. [15]. A mesoscopic generalization of the Lee-Yang lattice gas model is a lattice, the cells of which contain "elementary" (with the smallest allowable sizes) droplets and bubbles, which were called $l$ - and $g$-fluctuons. The linear cell size is evaluated as the doubled correlation length of the direct correlation function introduced by Ornstein and Zernike [20]. Fluctuons have a short average lifetime and mutually transform into one another. The sets of fluctuons of the same type form liquid-like and gas-like fractions of the fluid (the $l$ and $g$-fractions, respectively). Two adjacent fluctuons of different types repulse each other under the action of surface tension forces.

Assuming fluctuons to be statistically independent entities, let us take the chemical potential $\mu$ of the heterophase fluid in the form [15]

$\mu\left(\sigma^{(l)}, P, T\right)=\frac{1}{N} G\left(\sigma^{(l)}, P, T\right)=c^{(l)} \mu^{(l)}(P, T)+$

$+\left(1-c^{(l)}\right) \mu^{(g)}(P, T)+\mu_{\operatorname{mix}}\left(\sigma^{(l)}, P, T\right)$,

where

$\mu_{\text {mix }}\left(\sigma^{(l)}\right)=g_{2} \cdot \sigma^{(l)}\left(1-\sigma^{(l)}\right)+$

$+T \cdot\left[\sigma^{(l)} \ln \sigma^{(l)}+\left(1-\sigma^{(l)}\right) \ln \left(1-\sigma^{(l)}\right)\right]$.

Here, the superscripts $(l)$ and $(g)$ denote the quantities associated with the liquid and gaseous fractions, respectively; $N$ is the number of molecules; $G$ the free Gibbs energy; and $N^{(l, g)}, c^{(l, g)}=N^{(l, g)} / N$, and $\mu^{(l, g)}$ are the numbers, concentrations, and chemical potentials of molecules in the liquid $(l)$ and gaseous $(g)$ fractions. It is assumed that fluctuons of the $(l, g)$-th type contain $k^{(l, g)}$ molecules, and the quantities $\sigma^{(l, g)}$ are their concentrations: $\sigma^{(l, g)}=N_{f}^{(l, g)} / N_{f}$, where $N_{f}^{(l, g)}=N^{(l, g)} / k^{(l, g)}$ and $N_{f}=N_{f}^{(l)}+N_{f}^{(g)}$. The parameter $g_{2}$ is the constant of the interaction between the fluctuons of different types.

As one can see, the term $\mu_{\text {mix }}\left(\sigma^{(l)}\right)$ [Eq. (10)] in the chemical potential (9) describes the contribution made by the interaction of fluctuons and the entropy of their mixing to the chemical potential of a fluid, and it is of the Ising type. The first two terms on the right-hand side of Eq. (9) describe the contributions of molecules of the liquid and gaseous fractions to $\mu$. They are regular in the fluctuation region.

Changing to the variable $\alpha=\sigma^{(l)}-1 / 2$ in Eq. (9), one can observe that the CXC branches $\alpha_{\mathrm{CXC}}(\tau)$ determined by stable solutions of the equation of state $\partial \mu(\alpha, P, \tau) / \partial \alpha=0$ are symmetric, $\alpha_{\mathrm{CXC}}^{(l)}(\tau)=$ 

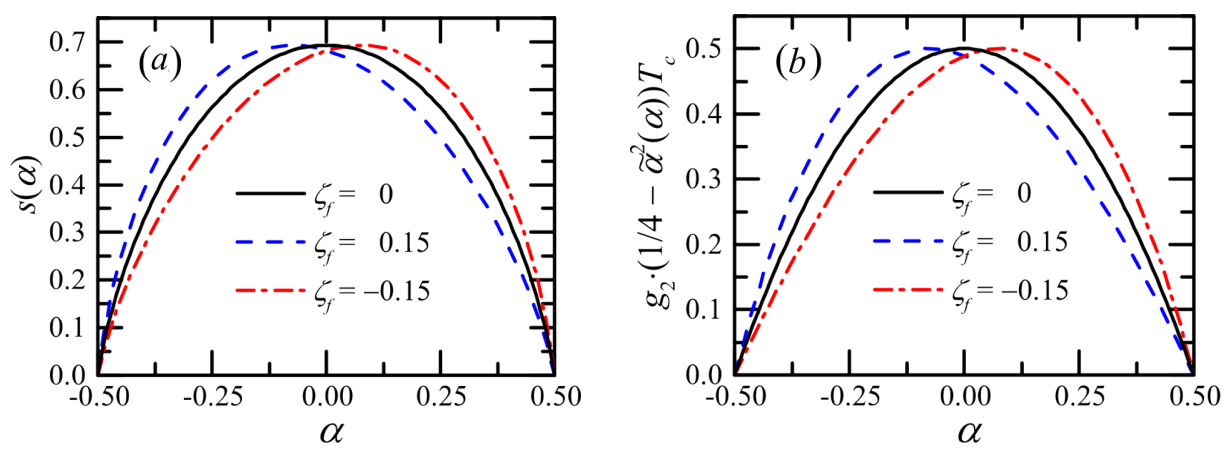

Fig. 1. Dependences of the mixing entropy $(a)$ and the pair interaction of fluctuons $(b)$ on the parameter $\alpha$ for some values of the asymmetry parameter $\zeta_{f}$

$=-\alpha_{\mathrm{CXC}}^{(g)}(\tau)$, with $\alpha_{\mathrm{c}}=0$ at the critical point. This result is a consequence of the fact that the volumes of all cells are equal.

In Ref. [15], the mesoscopic lattice model was generalized taking into account that, in effect, the volumes of $l$ - and $g$-fluctuons in a heterophase fluid are different. A transition to the "melted" lattice can be made with the help of the following nonlinear substitution of $\sigma$ by $\tilde{\sigma}$ :

$\tilde{\sigma}^{(i)}=\frac{\sigma^{(i)} w^{(i)}}{\sigma^{(l)} w^{(l)}+\sigma^{(g)} w^{(g)}} \quad(i=l, g)$

$w^{(l)}+w^{(g)}=1$.

Then the symmetry of the CXC $\alpha_{\mathrm{CXC}}(\tau)$ disappears. The corresponding expression for the chemical potential reads

$\mu(\tilde{\alpha}, P, T)=\bar{\mu}(P, T)-\frac{4 \tilde{\alpha}+\tilde{\zeta}}{4(1+\tilde{\alpha} \tilde{\zeta})} \Delta \mu(P, T)+$

$+\frac{T_{\mathrm{c}}}{\bar{k}} \frac{2-\zeta_{f} \tilde{\zeta}}{2\left(1-\zeta_{f}^{2}\right)} \frac{1+2 \zeta_{f} \tilde{\alpha}}{1+\tilde{\alpha} \tilde{\zeta}} \times\left[\frac{1}{2}-2 \tilde{\alpha}^{2}+(1+\tau) \times\right.$

$\left.\times\left(\frac{1}{2} \ln \left(\frac{1}{4}-\tilde{\alpha}^{2}\right)-\tilde{\alpha} \ln \left(\frac{1-2 \tilde{\alpha}}{1+2 \tilde{\alpha}}\right)\right)\right]$,

where the equality $g_{2}=2 T_{\mathrm{c}}$ for this chemical potential was taken into account, and the following notations were introduced:

$\bar{\mu}=\frac{\mu^{(g)}+\mu^{(l)}}{2}, \quad \Delta \mu=\mu^{(g)}-\mu^{(l)}$,

$\bar{k}=\frac{k^{(l)}+k^{(g)}}{2}, \quad \zeta=\frac{k^{(l)}-k^{(g)}}{\bar{k}}$,

$\tilde{\zeta}=\frac{2\left(\zeta+2 \zeta_{f}\right)}{2+\zeta \zeta_{f}}, \quad \tilde{\alpha}=\tilde{\sigma}^{(l)}-\frac{1}{2}$.

ISSN 2071-0194. Ukr. J. Phys. 2020. Vol. 65, No. 9
The difference

$\zeta_{f}=w^{(g)}-w^{(l)}$

is a parameter of asymmetry.

Note that this method allowing one to break the symmetry of $\alpha_{\mathrm{CXC}}(\tau)$ is only one among other possible variants.

Figure 1 demonstrates the dependences of the mixing entropy and the pair interaction of fluctuons on the parameter $\alpha$ for various values of the asymmetry parameter $\zeta_{f}$. Despite insignificant quantitative changes in the fluid thermodynamics, this asymmetry, as will be shown below, plays a crucial role at the determination of the coefficient $b_{2}$ and the amplitude $D_{1-\alpha}$.

\section{Amplitudes $D_{2 \beta}$ and $D_{1-\alpha}$ in Terms of the Mesoscopic Asymmetry Parameters}

The expressions for the series coefficients (7), which were found in Ref. [15], are given in Appendix. They are presented in terms of the mesoscopic asymmetry coefficients $\zeta_{f}, \zeta$, and $\tilde{\zeta}$. According to the SCS equations (8) and (A.1)-(A.4), we obtain the following expressions for the coefficients $a_{3}$ and $b_{2}$ :

$\frac{a_{3}}{1+a_{3}}=-\frac{4\left(\zeta-\zeta_{v c}\right)}{\zeta_{v c}(4-\tilde{\zeta} \zeta)}+\frac{16}{3} \frac{\left(\zeta-\zeta_{v c}\right)^{2}}{\zeta_{v c}^{2}\left(4-\tilde{\zeta}^{2}\right)^{2}} D_{0}$,

$b_{2}=2 \bar{k} \frac{1-\zeta_{f}^{2}}{2-\zeta_{f} \tilde{\zeta}}\left(\zeta_{f} \zeta_{v c} \frac{4-\tilde{\zeta}^{2}}{4-\zeta_{v c} \tilde{\zeta}}+D_{0}\right)$

When estimating the values of those parameters, the parameter $D_{0}$ can be neglected, because it is associated with the coefficients of linear thermal expansion and compressibility of $l$ - and $g$-fluctuons, which 

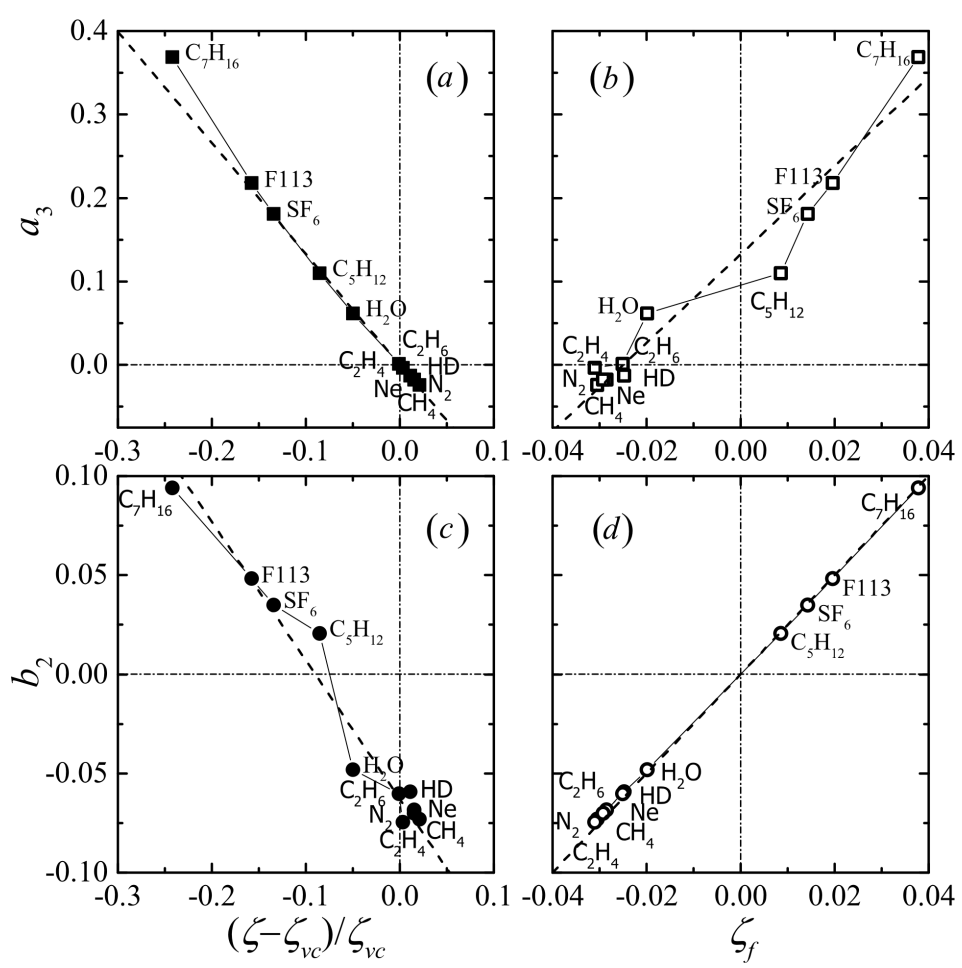

Fig. 2. Dependences of the parameters $a_{3}(a, b)$ and $b_{2}(c, d)$ on the mesoscopic asymmetry parameters $\zeta$ and $\zeta_{f}$ reproduced according to the data of Ref. [14]. The calculation parameters $\zeta_{v c}=0.8$ and $\bar{k}=3$

are non-singular in the critical region. As a result, we obtain

$$
\begin{aligned}
& \frac{a_{3}}{1+a_{3}} \cong-\frac{4\left(\zeta-\zeta_{v c}\right)}{\zeta_{v c}(4-\tilde{\zeta} \zeta)} \\
& b_{2} \cong 2 \bar{k} \zeta_{f} \zeta_{v c} \frac{1-\zeta_{f}^{2}}{2-\zeta_{f} \tilde{\zeta}}\left(\frac{4-\tilde{\zeta}^{2}}{4-\zeta_{v c} \tilde{\zeta}}\right)
\end{aligned}
$$

In this approximation, the parameter $b_{2}$ is proportional to the asymmetry parameter $\zeta_{f}$. One can see that the parameters $a_{3}$ and $b_{2}$ substantially depend on the mesoscopic asymmetry coefficients $\zeta$ and $\zeta_{f}$.

For the amplitudes $D_{2 \beta}$ and $D_{1-\alpha}$, we obtain the expressions

$$
\begin{aligned}
& D_{1-\alpha} \cong-\zeta_{f} \frac{3 \zeta_{v c}}{2} \frac{4-\tilde{\zeta}^{2}}{4-\tilde{\zeta} \zeta_{v c}}, \\
& D_{2 \beta} \cong-\frac{3 \zeta_{v c}(4-\tilde{\zeta})\left(\tilde{\zeta}-\zeta_{v c}\right)}{\left(4-\tilde{\zeta} \zeta_{v c}\right)^{2}}+\zeta_{f} \frac{3 \zeta_{v c}}{2} \frac{4-\tilde{\zeta}^{2}}{4-\tilde{\zeta} \zeta_{v c}} .
\end{aligned}
$$

It is of interest to know if there are master curves for the parameters $a_{3}$ and $b_{2}$ in terms of the mesoscopic asymmetry parameters. According to the values of those quantities determined in Ref. [14] for a number of fluids, we fitted the parameters $\zeta$ and $\zeta_{f}$ at the fixed values of the parameters $\bar{k}$ and $\zeta_{v c}$. In so doing, on the basis of estimates of the quantity $\bar{k}$ for simple liquids [22], we took $\bar{k}=3$. The parameter $\zeta_{v c}$ was taken to equal 0.8 . This choice corresponds to a density ratio of about 2.5:1 between the liquid and gas fractions.

The plots depicted in Fig. 2 illustrate the relation between the experimental data for the parameters $a_{3}$ and $b_{2}$ and the mesoscopic asymmetry parameters $\zeta$ and $\zeta_{f}$. The most demonstrative master curves are the straight line $a_{3} \approx-1.33\left(\zeta-\zeta_{v c}\right) / \zeta_{v c}$ in Fig. 2, $a$, which shows the dependence of the parameter $a_{3}$ on $\zeta$, and the straight line $b_{2} \approx 2.5 \zeta_{f}$ in Fig. $2, d$, which exhibits the dependence of the parameter $b_{2}$ on $\zeta_{f}$. Note that the dependences $a_{3}\left(\zeta_{f}\right)$ and $b_{2}(\zeta)$ are also approximately linear.

\section{Discussion and Conclusions}

The contribution of heterophase fluctuations to the thermodynamics of the critical fluid was considered, in that or another form, in a number of works (in ad- 
dition to those cited above, see also Refs. [23-25]) in the Frenkel approximation, i.e. assuming that the nuclei of the coexisting phase do not mutually interact.

It should be noted that van der Waals was the first who understood that just the appearance of heterogeneous unstable molecular formations (which he called pseudoassociations) in the fluid near the critical point is responsible for the deviation of the critical compressibility factor $Z_{\mathrm{c}}=P_{\mathrm{c}} v_{\mathrm{c}} / T_{\mathrm{c}}$ from a universal value of 0.375 obtained from the equation of corresponding states. In his Nobel Lecture [26], he paid considerable attention to elucidate the nature and role of pseudoassociations (associations of molecules without chemical bonds) at the qualitative level. He noted that a pseudoassociation, being a temporary object, contains densely packed molecular complexes [26]:

"Let the number of molecules that have combined into a complex be so large that it is possible to speak of a molecule at the center surrounded by a single layer containing almost as many other molecules as is possible simultaneously. Then, for the surrounding molecules the attraction directed towards the interior acts only to maintain the complex; and this part of its attraction is lost for the surface pressure. Only the forces acting outwards from these molecules can contribute to the formation of the internal pressure. But of course, for pseudo association as for true association the number of formed complexes increases with decreasing temperature and volume. At the critical point, so I was compelled to conclude, only a very small part of the weight is present as complexes. If pseudo association exists in a substance, there are at least two types of molecules, namely simple and complex."

However, van der Waals did not consistently examine the equation of fluid state in the presence of pseudoassociations and their contribution to the critical compressibility factor. To some extent, this consideration was done in Ref. [22]. Pseudoassociations of Van der Waals can be regarded as a prototype of Frenkel's heterophase fluctuations. As concerning his assumption that, near the critical point, only an insignificant fraction of molecules belongs to molecular complexes, it is not justified, because the critical point lies in the region of strong heterophase fluctuations.

Kadanoff marked [20] that, at scaling transformations, the block size has to be confined from below by the size of the region of short-range molecular correlations, in which the homogeneity of scaling transformations becomes lost. The "interaction volume" $\varpi^{*}$, which was used in Refs. $[13,14]$ at the ordering of the parameters $a_{3}$ and $b_{2}$, is an average microscopic quantity that characterizes the local interaction of a molecule with its environment. Instead, the volumes of fluctuons are mesoscopic parameters. The fact that these volumes are not identical in the general case results in the mixing of the quantities $\sigma^{(l)}$ and $\sigma^{(g)}$ [see Eq. (11)] and the appearance of the asymmetry parameter $\zeta_{f}$.

Experimental results testify that the leading singular components of the CXC diameter for liquids of various nature have a universal form [see Eq. (3)]. This is also true for substances for which there coexist two or more types of short-range correlations in the liquid, and the gas-liquid transition is accompanied by transformations associated with the evolution of the short-range ordering of molecules. For instance, mercury belongs to such substances. More specifically, the liquid-like fraction of mercury is a mixture of metallic and semiconducting fluctuons, so that the gas-liquid transition is accompanied by the insulatorsemiconductor-metal transformations [27]. It is evident that, in this case, the number of mesoscopic asymmetry parameters in the effective Hamiltonian increases with the number of contributing shortrange-order types.

The answer to the question "How effective is the SCS approach for finding the amplitudes $D_{2 \beta}$ and $D_{1-\alpha}$ in the case where the number of asymmetry parameters increases?" is reduced to finding the series coefficients $\mu_{m n}$ which determine the parameters $a_{3}$ and $b_{2}$ in Eqs. (8). In Ref. [27], we estimated the most probable values of the parameters $a_{3}$ and $b_{2}$. For this purpose, we fitted the amplitudes $D_{2 \beta}$ and $D_{1-\alpha}$ by varying the "effective" values of the asymmetry parameters $\zeta$ and $\zeta_{f}$. However, the problem of finding the coefficients $\mu_{m n}$ for complex fluids remains open.

In order to compare our results of the ordering of the parameters $a_{3}$ and $b_{2}$ with those proposed in Refs. $[13,14]$ at a qualitative level, let us plot the master curves $a_{3}\left(\varpi^{*}\right)$ and $b_{2}\left(\varpi^{*}\right)$ from those works (see Fig. 3) and compare them with ours (Fig. 2). One can see that the relation of the parameters $a_{3}$ and $b_{2}$ with $\varpi^{*}$ is rather unambiguous at $\varpi^{*}<0.2$, which corresponds to fluids with large molecular weights. At $\varpi^{*}>0.2$, the unambiguity disappears. At the same 

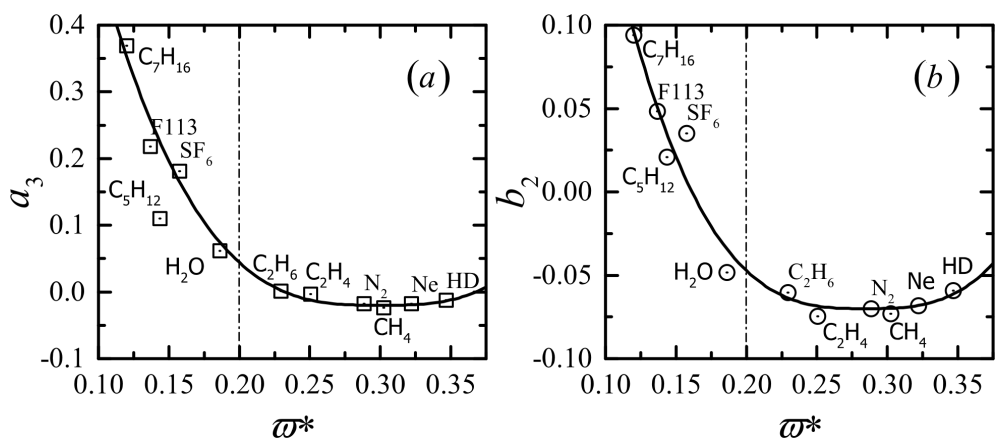

Fig. 3. Asymmetry coefficients $a_{3}$ and $b_{2}$ of the complete scaling model as functions of "interaction volume" $\varpi *$. According to the data of Refs. [13, 14]

time, the linear master curves in Figs. 2, $a$ and $d$ acceptably put into order the parameters $a_{3}$ and $b_{2}$ determined from the analysis of experimental data. This circumstance gives us the reason to conclude that the mesoscopic asymmetry parameters are responsible for the amplitudes $D_{2 \beta}$ and $D_{1-\alpha}$ of the leading singular components of the CXC diameter. As an example, here are the values of the mesoscopic asymmetry parameters $\zeta$ and $\zeta_{f}$ determined using the linear master curves (Fig. 2) for ethane: $\zeta=0.788$ and $\zeta_{f}=-0.025$.

The authors are grateful to L.A. Bulavin for valuable discussion.

\section{APPENDIX}

The expressions for $\mu_{m n}$ in terms of the mesoscopic model parameters [15] look like

$$
\begin{aligned}
& \mu_{11}=\frac{2}{\bar{k} \zeta_{v c}^{2}} \frac{2-\tilde{\zeta} \zeta_{f}}{1-\zeta_{f}^{2}}\left(\frac{4-\tilde{\zeta} \zeta_{v c}}{4-\tilde{\zeta}^{2}}\right)^{2}+O\left(\bar{k}^{-2}\right) \\
& \mu_{21}=\frac{4}{\bar{k} \zeta_{v c}^{3}} \frac{2-\tilde{\zeta} \zeta_{f}}{1-\zeta_{f}^{2}}\left(\frac{4-\tilde{\zeta} \zeta_{v c}}{4-\tilde{\zeta}^{2}}\right)^{3} \times \\
& \times\left(3 \zeta_{f}+6 \frac{\tilde{\zeta}-\zeta_{v c}}{4-\tilde{\zeta} \zeta_{v c}}+\frac{4}{\zeta_{v c}} \frac{4-\tilde{\zeta} \zeta_{v c}}{4-\tilde{\zeta}^{2}} D_{0}\right)+O\left(\bar{k}^{-2}\right), \\
& \mu_{30}=\frac{16}{\bar{k} \zeta_{v c}^{4}} \frac{2-\tilde{\zeta} \zeta_{f}}{1-\zeta_{f}^{2}}\left(\frac{4-\tilde{\zeta} \zeta_{v c}}{4-\tilde{\zeta}^{2}}\right)^{4}+O\left(\bar{k}^{-2}\right) \\
& \mu_{40}=\frac{160}{\bar{k} \zeta_{v c}^{5}} \frac{2-\tilde{\zeta} \zeta_{f}}{1-\zeta_{f}^{2}}\left(\frac{4-\tilde{\zeta} \zeta_{v c}}{4-\tilde{\zeta}}\right)^{4} \times \\
& 6\left(\tilde{\zeta}-\zeta_{v c}\right)+\zeta_{f}\left(4-\tilde{\zeta} \zeta_{v c}\right) \\
& \times \frac{\tilde{\zeta}^{2}}{\left.4-\bar{k}^{-2}\right)}
\end{aligned}
$$

where

$D_{0}=T_{\mathrm{c}}\left(\bar{\kappa}_{\mathrm{c}}-\frac{\tilde{\zeta}-\zeta_{v c}}{4-\tilde{\zeta} \zeta_{v c}} \Delta \kappa_{\mathrm{c}}\right)-$

\section{8}

$$
\begin{aligned}
& -\frac{4-\tilde{\zeta} \zeta_{v c}}{4 \zeta_{v c}} \frac{\Delta s_{\mathrm{c}} P_{\mathrm{c}}}{Z_{\mathrm{c}}}\left(\bar{\beta}_{\mathrm{c}}-\frac{\tilde{\zeta}-\zeta_{v c}}{4-\tilde{\zeta} \zeta_{v c}} \Delta \beta_{\mathrm{c}}\right), \\
& \bar{\kappa}_{\mathrm{c}}=\frac{\kappa_{\mathrm{c}}^{(g)}+\kappa_{\mathrm{c}}^{(l)}}{2}, \quad \Delta \kappa_{\mathrm{c}}=\kappa_{\mathrm{c}}^{(g)}-\kappa_{\mathrm{c}}^{(l)}, \\
& \Delta s_{\mathrm{c}}=s_{\mathrm{c}}^{(g)}-s_{\mathrm{c}}^{(l)}, \\
& \bar{\beta}_{\mathrm{c}}=\frac{\beta_{\mathrm{c}}^{(g)}+\beta_{\mathrm{c}}^{(l)}}{2}, \quad \Delta \beta_{\mathrm{c}}=\beta_{\mathrm{c}}^{(g)}-\beta_{\mathrm{c}}^{(l)} .
\end{aligned}
$$

Here, $s_{\mathrm{c}}^{(g, l)}, \kappa_{\mathrm{c}}^{(g, l)}$, and $\beta_{\mathrm{c}}^{(g, l)}$ are the specific entropy, the coefficient of thermal expansion, and the compressibility of the gaseous $(g)$ and liquid $(l)$ fractions of the fluid at the critical point, respectively.

1. J. Weiner, K.H. Langley, N.C. Ford, Jr. Experimental evidence for a departure from the law of the rectilinear diameter. Phys. Rev. Lett. 32, 879 (1974).

2. M. Ley-Koo, M. S. Green. Revised and extended scaling for coexisting densities of $\mathrm{SF}_{6}$. Phys. Rev. A 16, 2483 (1977).

3. L. Bulavin, Yu. Shimanskii, Singularity of the diameter of the coexistence curve of ethane. JETP Lett. 29, 438 (1979).

4. E.T. Shimanskaya, I.V. Bezruchko, V.I. Basok, Yu.I. Shimanskii. Experimental determination of the critical exponent and of the asymmetric and nonasymptotic corrections to the equation of the coexistence curve of Freon-113. Sov. Phys. JETP 53, 139 (1981).

5. S. Jüngst, B. Knuth, F. Hensel. Observation of singular diameters in the coexistence curves of metals. Phys. Rev. Lett. 55, 2160 (1985).

6. L.P. Kadanoff. Scaling laws for Ising models near $T_{\mathrm{c}}$. Physics 2, 263 (1966).

7. K.G. Wilson, J. Kogut. The renormalization group and the $\epsilon$ expansion. Phys. Rep. 12, 75 (1974).

8. K.G. Wilson. The renormalization group: Critical phenomena and the Kondo problem. Rev. Mod. Phys. 47, 773 (1975).

9. I.R. Yukhnovskii, M.F. Golovko. Statistical Theory of Classical Equilibrium Systems (Naukova Dumka, 1987) (in Russian).

ISSN 2071-0194. Ukr. J. Phys. 2020. Vol. 65, No. 9 
10. T.D. Lee, C.N. Yang. Statistical theory of equations of state and phase transitions. II. Lattice gas and Ising model. Phys. Rev. 87, 410 (1952).

11. M.E. Fisher, G. Orkoulas. The Yang-Yang anomaly in fluid criticality: experiment and scaling theory. Phys. Rev. Lett. 85, 696 (2000).

12. Y.C. Kim, M.E. Fisher, G. Orkoulas. Asymmetric fluid criticality. I. Scaling with pressure mixing. Phys. Rev. E 67, 061506 (2003).

13. M.A. Anisimov, J. Wang. Nature of asymmetry in fluid criticality. Phys. Rev. Lett. 97, 025703 (2006).

14. J. Wang, M.A. Anisimov. Nature of vapor-liquid asymmetry in fluid criticality. Phys. Rev. E 75, 051107 (2007).

15. O. Bakai. Mesoscopic equation of state of the heterophase fluid and its application to description of the liquid-gas asymmetry. J. Mol. Liq. 235, 135 (2017).

16. R.A. Perkins, J.V. Sengers, I.M. Abdulagatov, M.L. Huber. Simplified model for the critical thermal-conductivity enhancement in molecular fluids. Int. J. Thermophys. 34, 191 (2013).

17. J. Frenkel. A general theory of heterophase fluctuations and pretransition phenomena. J. Chem. Phys. 7, 538 (1939).

18. J.I. Frenkel. Kinetic Theory of Liquids (Oxford Univ. Press, 1946)

19. V.I. Yukalov. Phase transitions and heterophase fluctuations. Phys. Rep. 208, 395 (1991).

20. L.P. Kadanoff. Relating theories via renormalization. Stud. Hist. Philos. Sci. B 44, 22 (2013).

21. L.S. Ornstein, F. Zernike. Accidental deviations of density and opalescence at the critical point of a single substance. Proc. Sect. Sci. K. Ned. Akad. Wet. 17, 793 (1914).

22. O. Bakai. The van der Waals idea of pseudo associations and the critical compressibility factor. Cond. Mat. Phys. 23, 13603 (2020).
23. M.E. Fisher. The theory of condensation and the critical point. Physics 3, 255 (1967).

24. K.S. Kiang, D. Stauffer. Application of Fisher's droplet model for liquid-gas transition near $T_{\mathrm{c}}$. Z . Phys. A $\mathbf{2 3 5}$, 130 (1970).

25. I.R. Yukhnovskyi. Phase transitions near the gas-liquid critical point. Ukr. Fiz. Zh. Oglyady 10, 33 (2015) (in Ukrainian).

26. J.D. van der Waals. The equation of state for gases and liquids. In: Nobel Lectures, Physics 1901-1921 (Elsevier, 1967), p. 254.

27. O. Bakai, M. Bratchenko, S. Dyuldya. Three-state mesoscopic model of a heterophase fluid in application to the gas-liquid and dielectric-semiconductor-metal transformations in expanded mercury. J. Mol. Liq. 260, 245 (2018).

Received 13.04.20.

Translated from Ukrainian by O.I. Voitenko

\section{О. Бакай, М. Братченко, С. Дюлъдя}

ПРО СИНГУЛЯРНІСТЬ ДІАМЕТРА

КРИВОЇ СПІВІСНУВАННЯ РІДИНА-ГАЗ

$\mathrm{P}$ е $з$ ю м е

Метод спрощеного "повного" скейлінгу Анісімова-Ванга дозволяє знайти амплітуди сингулярностей діаметра кривої співіснування (KC) фаз за коефіцієнтами розкладання середньопольової вільної енергії за ступенями приведених температури і густини в околі критичної точки. Цей метод застосовано при визначенні амплітуд головних критичних сингулярностей діаметра $\mathrm{KC}$ для плину, що описується мезоскопічною теорією. Згідно з одержаними результатами, амплітуди головних сингулярностей діаметра $\mathrm{KC}$ визначаються мезоскопічними параметрами асиметрії гетерофазного плину. 